EPJ Web of Conferences 73, 05008 (2014)

DOI: $10.1051 /$ epjconf/20147305008

(C) Owned by the authors, published by EDP Sciences, 2014

\title{
X-ray spectroscopy of kaonic atoms at SIDDHARTA
}

\author{
M. Cargnelli ${ }^{1, \mathrm{a}}$, M. Bazzi ${ }^{2}$, G. Beer ${ }^{3}$, C. Berucci ${ }^{1,2}$, A.M. Bragadireanu ${ }^{1,5}$, C. Curceanu ${ }^{2}$, A. d'Uffizi ${ }^{2}$, \\ C. Fiorini ${ }^{4}$, F. Ghio ${ }^{6}$, C. Guaraldo ${ }^{2}$, R. Hayano ${ }^{7}$, M. Iliescu ${ }^{2}$, T. Ishiwatari ${ }^{1}$, M. Iwasaki ${ }^{8}$, P. Levi

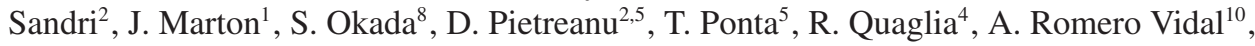 \\ E. Sbardella ${ }^{2}$, A. Scordo ${ }^{2}$, H. Shi ${ }^{1,7}$, D.L. Sirghi ${ }^{2,5}$, F. Sirghi ${ }^{2,5}$, H. Tatsuno ${ }^{2}$, O. Vazquez Doce ${ }^{9}$, \\ E. Widmann ${ }^{1}$, B. Wünschek ${ }^{1}$ and J. Zmeskal ${ }^{1}$
}

${ }^{1}$ Stefan-Meyer-Institut für subatomare Physik, Boltzmanngasse 3, 1090 Wien, Austria ${ }^{2}$ INFN, Laboratori Nazionali di Frascati, C.P. 13, via E. Fermi 40, 00044 Frascati (Roma), Italy ${ }^{3}$ Dep. of Physics and Astronomy, University of Victoria, PO Box 3055, Victoria B.C. Canada V8W3P6 ${ }^{4}$ Politecnico di Milano, Dip. di Elettronica e Informazione, Piazza L. da Vinci 32, 20133 Milano, Italy ${ }^{5}$ Institutul National pentru Fizica si Inginerie Nucleara Horia Hulubei, Reactorului 30, Magurele, Romania

${ }^{6}$ INFN Sez. di Roma I and Instituto Superiore di Sanita 00161, Roma, Italy

${ }^{7}$ University of Tokyo,7-3-1, Hongo, Bunkyo-ku,Tokyo, Japan

${ }^{8}$ RIKEN, Institute of Physical and Chemical Research, 2-1 Hirosawa, Wako, Saitama 351-0198, Japan ${ }^{9}$ Excellence Cluster Universe, Tech. Univ. München, Boltzmannstraße 2, 85748 Garching, Germany

${ }^{10}$ Universidade de Santiago de Compostela, Casas Reais 8, 15782 Santiago de Compostela, Spain

\begin{abstract}
The X-ray measurements of kaonic atoms play an important role for understanding the low-energy QCD in the strangeness sector. The SIDDHARTA experiment studied the X-ray transitions of 4 light kaonic atoms $\left(\mathrm{H}, \mathrm{D},{ }^{3} \mathrm{He}\right.$, and $\left.{ }^{4} \mathrm{He}\right)$ using the DAFNE electron-positron collider at LNF (Italy). Most precise values of the shift and width of the kaonic hydrogen 1s state were determined, which have been now used as fundamental information for the low-energy $K^{-} \mathrm{p}$ interaction in theoretical studies. An upper limit of the X-ray yield of kaonic deuterium was derived, important for future $K^{-} \mathrm{d}$ experiments. The shifts and widths of the kaonic ${ }^{3} \mathrm{He}$ and ${ }^{4} \mathrm{He} 2 \mathrm{p}$ states were obtained, confirming the end of the "kaonic helium puzzle". In this contribution also the plans for new experiments of kaonic deuterium are being presented.
\end{abstract}

\section{Introduction}

The strong interaction between hadrons at low energies e.g., between the antikaon and the nucleon $(\bar{K} N)$ cannot be described in terms of quarks and gluons. Instead, effective field theories are used which rely on experimental input. The data in the strangeness sector come from kaon scattering experiments, atomic X-ray measurements and the energies, widths and branching ratios of known resonances.

\footnotetext{
ae-mail: Michael.Cargnelli@oeaw.ac .at
}

This is an Open Access article distributed under the terms of the Creative Commons Attribution License 4.0, which permits unrestricted use, distribution, and reproduction in any medium, provided the original work is properly cited. 
SIDDHARTA

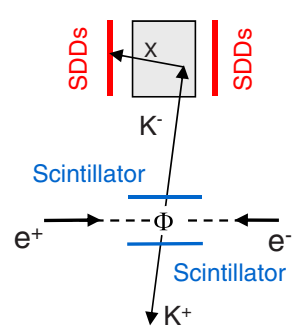

SIDDHARTA-2

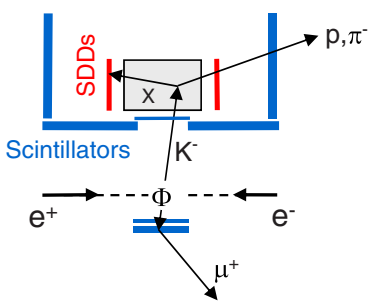

Kd.JPARC

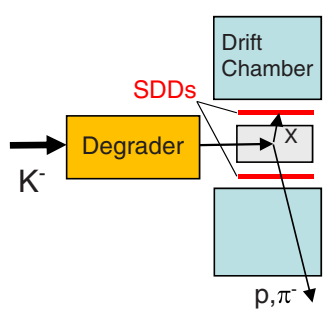

Figure 1. Sketch of the setups and experimental methods. Left: SIDDHARTA, Middle: SIDDHARTA-2, note the changed position of the upper trigger scintillator and the additional scintillators used to detect kaon secondaries and beam-background. Right: possible alternative experiment at J-PARC, Japan

Table 1. Compilation of SIDDHARTA results. The errors given in this table are the sum of the statistical and the systematic component; in case of asymmetric errors, the larger one is quoted here. The upper limits of the yields are for CL 90\%. The yields for $\mathrm{H}$ and $\mathrm{He}$ are preliminary values. Gas densities: H: $14.5 \rho_{S T P}$, D: $\left.13.9 \rho_{S T P}\right),{ }^{3} \mathrm{He}$ : $5.38 \rho_{S T P},{ }^{4} \mathrm{He}: 9.24 \rho_{S T P}$. For kaonic carbon etc. see [6].

\begin{tabular}{ccccc}
\hline & shift $[\mathrm{eV}]$ & width $[\mathrm{eV}]$ & transition yields in \% per stopped $K^{-}$ & ref. \\
\hline $\mathrm{H}$ & $\epsilon_{1 s}=-283 \pm 42$ & $\Gamma_{1 s}=541 \pm 111$ & $\mathrm{Y}\left(K_{\text {tot }}\right)=4.5 \pm 1.2 \mathrm{Y}\left(K_{\alpha}\right)=1.2 \pm 0.4$ & {$[7-9]$} \\
$\mathrm{D}$ & & & $\mathrm{Y}\left(K_{\text {tot }}\right)<1.43 \mathrm{Y}\left(K_{\alpha}\right)<0.39$ & {$[10]$} \\
${ }^{3} \mathrm{He}$ & $\epsilon_{2 p}=-2 \pm 6$ & $\Gamma_{2 p}=6 \pm 13$ & $\mathrm{Y}\left(L_{\alpha}\right)=25.0 \pm 6.7$ & {$[11,13,14]$} \\
${ }^{4} \mathrm{He}$ & $\epsilon_{2 p}=5 \pm 7$ & $\Gamma_{2 p}=14 \pm 13$ & $\mathrm{Y}\left(L_{\alpha}\right)=23.1 \pm 6.0$ & {$[12-14]$} \\
\hline
\end{tabular}

The kaonic atom experiments extract the effect of the strong interaction on the low-lying states by measuring X-ray transitions to these levels, seen as a shift and broadening with respect to the QED calculated values. For current theoretical work see [1,2] and references therein.

The $\bar{K} N$ interaction at rest is normally described in terms of complex scattering lengths. To extract the isospin $(\mathrm{I}=0,1)$ dependent $\bar{K} N$ scattering lengths, $a_{0}$ and $a_{1}$, measurements of kaonic deuterium are necessary along with kaonic hydrogen data.

In the case of kaonic ${ }^{4} \mathrm{He}$ the $2 \mathrm{p}$ shift measured in $[3,4]$ was $-43 \pm 8 \mathrm{eV}$ on average, whereas theoretical calculations gave a shift below $1 \mathrm{eV}$ ("kaonic helium puzzle"). The first experimental result consistent with theory was [5]. Within SIDDHARTA the zero-compatible shift was confirmed in a measurement with other systematics and also with ${ }^{3} \mathrm{He}$.

\section{The SIDDHARTA experiment}

Figure 1 (left) illustrates the principle of the SIDDHARTA experiment. Both kaons of a charged kaon pair are detected and define the trigger. Kaonic atoms are formed when negative kaons $\left(K^{-}\right)$enter the gas in the target cell and loose their remaining kinetic energy through ionization and excitation and, by replacing an electron, are captured in an excited orbit. Via different cascade processes, the kaonic atoms deexcite to lower states. When the $K^{-}$reach low-n states with small angular momentum, they are absorbed by the nucleus. A small fraction of these kaonic atoms survive long enough for one of the (np-1s) transitions (K-series). The X-rays of these transitions are detected by 144 Silicon Drift Detectors (SDDs) placed around the target. Hits in the X-ray detectors coincident with the trigger contain kaonic $\mathrm{X}$-rays along with background from particles generated by kaon decay and $K^{-}$absorption. Due to the limited timing resolution some accidental (not kaon-correlated) background also remains. For a more detailed description see [7,8]. Table 1 gives a compilation of SIDDHARTA results. Note that the 
transition yields depend on the target density and also on the strong interaction parameters (the shift and width of the lower, and the width of the upper state) and deliver information on the cascade and crosscheck the measured shift and width.

\section{Future kaonic deuterium experiments}

A quantitative measurement of kaonic deuterium X-rays requires a substantial improvement of the applied experimental technique. Since the yield is expected to be about $1 / 10$ of the KH yield and the width up to twice the KH width, we need a reduction in background of a factor about 20 and an increase in the detection efficiency of 2-3. For the simulations we use a yield $\mathrm{Y}\left(K_{\alpha}\right)=0.001$ derived from predictions [15-18]. The reliability of these cascade calculations can be estimated to be better then to a factor of 2, derived from our experiences from kaonic hydrogen [9]. The upgrade to SIDDHARTA-2 is based on four main modifications.

(1) Trigger geometry and target density: By placing the upper kaon-trigger detector close in front of the target entrance window, the probability that a triggered kaon really enters the gas and is stopped there is much improved. Making the detector smaller than the entry area gives away some signal, but suppresses efficiently the kaonic lines from "wallstops" (kaons entering the gas volume, but passing from the inside of the target to the cylindrical walls). The number "signal per trigger" goes up, which also reduces the accidental background coming along with every trigger. We plan as well to double the gas density which enhances the gas stops and further reduces the wall-stops.

(2) $\mathrm{K}^{+}$discrimination to suppress kaon decay background: A "kaon stopper" scintillator is placed directly below the lower kaon trigger scintillator. When a $K^{-}$is stopped there, only one (large) signal from pileup of stopping and kaon-absorption-secondaries is seen, whereas when a $K^{+}$is stopped, the kaon-decay particles are seen separately after the signal from the stopping (mean $K^{+}$lifetime $12.8 \mathrm{~ns}$ ). In addition, we will use scintillators surrounding the target to measure $\mathrm{K}^{-}$absorption secondaries (pions). The time window for gas stops is about $4 \mathrm{~ns}$ wide. By this condition we also suppress stops in the entry window.

(3) Active shielding: The scintillators surrounding the target will also be used in prompt anticoincidence if the spatial correlation of SDD and scintillator hits indicates that it originated from a pion ("charged particle veto"). An anticoincidence covering the SDD time window (with the exception of the $4 \mathrm{~ns}$ of the gas stopping time) will reduce the accidental background. Although the scintillators have only low efficiency for gammas, the abundance of secondaries from the electromagnetic showers allows a relevant reduction of accidental ("beam") background.

(4) Operating SDDs at a lower temperature: tests indicate that an improvement of the timing resolution by a factor of 1.5 is feasible by more cooling.

The signal enhancement by a factor 2 to 3 is due to moving the target cell closer to the IP, by changing its shape, by a better solid angle of the SDDs and by the higher gas density. In such conditions, with an integrated luminosity of $800 \mathrm{pb}^{-1}$ a precision of about $70 \mathrm{eV}$ for the shift, and $160 \mathrm{eV}$ for the width are attainable, resulting in a relative precision similar to that obtained for kaonic hydrogen.

Since the timeline at DAFNE is not set clearly, alternatively we studied the feasibility of a kaonic deuterium measurement at the 1.8BR beam line of J-PARC in Japan. See Table 2.

\section{Summary and conclusions}

The SIDDHARTA experiment has resulted in unprecedented measurements of light kaonic atoms. The strong-interaction induced shift and width of the ground state of kaonic hydrogen and of the $2 \mathrm{p}$ state of kaonic helium was measured along with the transition yields. Furthermore, we worked out a scheme for 


\section{EPJ Web of Conferences}

Table 2. Comparison of properties of future kaonic deuterium experiments.

\begin{tabular}{cc}
\hline SIDDHARTA-2 & Kd.JPARC \\
\hline $10 \mathrm{pb}^{-1}$ per day, 80 days & $30 \mathrm{KW}$ proton beam, 35 days \\
$1.5 \times 10^{7} \mathrm{~K}^{ \pm}$pairs per day. SOURCE & $4 \times 10^{7} \mathrm{~K}^{-}$per day. BEAM \\
$\mathrm{p}=127 \mathrm{MeV} / \mathrm{c} \mathrm{E}=16 \mathrm{MeV}$ & $\mathrm{p}=660 \mathrm{MeV} / \mathrm{c} \mathrm{E}=331 \mathrm{MeV}$ \\
$2 \%$ gasstops (small solid angle) & $0.03 \%$ gasstops (energy distribution, loss in degrader) \\
$144 \mathrm{~cm}^{2}$ SDDs from SIDDHARTA & $288 \mathrm{~cm}^{2}$ new improved SDDs \\
small absorption in flight & substantial in-flight contribution \\
stopping well localized & fiducial volume cut needed \\
equipment in advanced status & proposal in preparation \\
\hline
\end{tabular}

an improved experimental technique "SIDDHARTA-2" which promises to deliver a sound quantitative measurement of the shift and width in kaonic deuterium, long awaited for the description of the strong interaction at lowest energies in hadronic systems with strangeness.

\section{References}

[1] M. Döring, U.-G. Meißner, Phys. Lett. B 704, 663 (2011)

[2] Y. Ikeda, T. Hyodo, W. Weise, Phys. Lett. B, 706, 63 (2011)

[3] S. Baird, et al., Nucl. Phys. A 392, 297 (1983)

[4] C.J. Batty, et al., Nucl. Phys. A 508, 89 (1990)

[5] S. Okada, et al., Phys. Lett. B 653, 387 (2007)

[6] (SIDDHARTA Collaboration), Nucl. Phys. A 916, 30 (2013)

[7] (SIDDHARTA Collaboration), Phys. Lett. B 704, 113 (2011)

[8] (SIDDHARTA Collaboration), Nucl. Phys. A 881, 88 (2012)

[9] (SIDDHARTA Collaboration), EPJ Web of Conferences, Proc. of INPC 2013, in press

[10] (SIDDHARTA Collaboration), Nucl. Phys. A 907, 69 (2013)

[11] (SIDDHARTA Collaboration), Phys. Lett. B 697, 199 (2011)

[12] (SIDDHARTA Collaboration), Phys. Lett. B 681, 310 (2009)

[13] (SIDDHARTA Collaboration), Phys. Lett. B 714, 40 (2012)

[14] (SIDDHARTA Collaboration), EPJ A Letters, to be published

[15] M. Faber et al., Phys. Rev. C 84, 064314 (2011)

[16] T.S. Jensen, Frascati Physics Series Vol. XXXVI (2004)

[17] T. Koike, et al., Phys. Rev. C 53, 79-87 (1996)

[18] M. Raeisi G. and S.Z. Kalantari, Phys. Rev. A 79, 012510 (2009) 